\title{
The Influence of Atmospheric Turbulence on Radio Vortex Wave
}

\author{
Ling-Ling Li, Heng-Kai Zhao, Shao-Jun Zhang, Xue-Feng Liu \\ School of Communication and Information Engineering, Shanghai University, Shanghai, China \\ E-mail: LILI9408@163.com; hkzhao@ staff.shu.edu.cn
}

\begin{abstract}
Spiral space phase distribution of the vortex wave form specific orbital angular momentum. This paper studies the effect of atmospheric turbulence on the vortex wave phase through the numerical simulation. In order to analyze the influence of atmospheric turbulence on the vortex wave, we make comparison between various effects by different atmosphere structure constants, transmission distances and topological charges. When the refractive index structure constant is $8 \times 10^{-15} \mathrm{~m}^{-2 / 3}$, the spiral phase change is about $37.5 \%$; When the refractive index structure constant increases up to a value of $8 \times 10^{-13} \mathrm{~m}^{-2 / 3}$ accordingly, spiral phase increases to $50 \%$; when transmission distance reaches up to $1000 \mathrm{~m}$, phase change increases to $62.5 \%$. At the same time, in different azimuth space, spiral phase change effect is also different. The research results show that the strength of the atmospheric turbulence, space orientation, and wave transmission distance will make the OAM phase distribution of the vortex wave changes, therefore, they are the important factors of affecting the characteristics of the vortex waves OAM.
\end{abstract}

Keywords-orbital angular momentum; atmospheric turbulence; vortex wave; phase characteristic

\section{INTRODUCTION}

In order to improve the communication system capacity and spectrum efficiency, it's necessary to explore innovative technology. In recent years, orbital angular momentum $(\mathrm{OAM})$ becomes a study hotspot and is causing more and more widely concerned in wireless communication. However, the performance of communication system in free-space is inevitably affected by space propagation environment ${ }^{[10]}$. In this paper we analyze the influence of atmospheric turbulence on radio vortex wave signal phase, give the simulation of transmission signal phase under different transmission distance and different atmospheric turbulence intensity, and make comparison of different influences between the two topological charges of $l=1$ and $l=4$.

\section{THE MAIN FEATURES OF VORTEX WAVE ORBITAL ANGULAR MOMENTUM}

We can add a twiddle factor associated with spatial phase angle on the normal electromagnetic wave to generate vortex electromagnetic wave, the vortex electromagnetic waves can be represented as follows ${ }^{[8]}$ :

$$
U(r, \theta)=A(r) \times e^{i l \theta}
$$

In which, $A(r)$ is the amplitude of normal electromagnetic wave, $r$ is the radiation distance from the center of a beam, $\theta$ is space phase angle and $l$ is the intrinsic value of orbital angular momentum.

At the transmitter, the plane electromagnetic wave revolve and form vortex electromagnetic wave; at the receiver, in order to gain the normal electromagnetic waves, the vortex electromagnetic wave should rotate according to the opposite direction. OAM beams with different topological charges of $l$ values are mutually orthogonal, and they satisfy the following formula ${ }^{[3]}$ :

$$
\begin{aligned}
& \left(U_{1}(r, \theta), U_{2}(r, \theta)\right)=\int U_{1}(r, \theta) U_{2}^{*}(r, \theta) r d r d \theta \\
& =\left\{\begin{array}{l}
0, \forall l_{1} \neq l_{2} \\
\int\left|U_{1}(r, \theta)\right|^{2} r d r d \theta, \quad l_{1}=l_{2}
\end{array}\right.
\end{aligned}
$$

We can multiply OAM beams with different $l$ values, and embed these different electromagnetic OAM beams on the same frequency, therefore, improve the system capacity and spectrum efficiency.

In this paper, we use Laguerre-Gaussian (LG) beam to produce the OAM beams. The expression for the cylindrical coordinate of LG vortex waves which transmit along $Z$ axis about $z$ distance is:

$$
A(r, \phi, z)=R_{l, p}(r, z) \cdot e^{i l \phi} \cdot \exp \left\{-i(2 p+|l|+1) \cdot \tan ^{-1} \frac{z c}{\pi f w_{0}^{2}}\right\}
$$

where

$$
\begin{gathered}
R_{l, p}(r, z)=\frac{1}{w(z)} \cdot\left[\frac{2 p !}{\pi(|l|+p) !}\right]^{\frac{1}{2}} \times \\
{[\sqrt{2} r / w(z)] \cdot L_{p}^{l} \cdot\left(\frac{2 r^{2}}{w^{2}(z)}\right) \cdot e^{-\frac{r^{2}}{w^{2}(z)}} \cdot e^{-\frac{i \pi f r^{2}}{R c}}} \\
w(z)=w_{\mathrm{O}} \cdot \sqrt{1+\left(z c / \pi f w_{\mathrm{O}}^{2}\right)^{2}} \\
R=z\left[1+\left(\pi f w_{0}^{2} / z \cdot c\right)^{2}\right]
\end{gathered}
$$

In formula(3), $l$ is the intrinsic value of OAM, $p$, the index of radiation, $w_{0}$, the waist radius, $r$ is the radiation radius, $L_{P}|l|$, the Laguerre polynomials, $\Phi$, the radiation angle, $c$, the velocity of wave propagation, $f$, the frequency of the wave. 


\section{ATMOSPHERE STRUCTURE CONSTANT $C_{n}{ }^{2}$ OF MICROWAVE B AND}

About $C_{n}{ }^{2}$, ITU-R has presented its model, but this model is applicable for the band of light wave. For microwave band, we have to consider some new factors such as atmosphere relative humidity or water vapor content and other factors. In other words, an important factor influencing the microwave band $C_{n}{ }^{2}$ is the humidity in the air, so the microwave band $C_{n}{ }^{2}(T, R)$ is a function of temperature and humidity. As the elevation altering, temperature and humidity will change and therefore cause variation of $C_{n}{ }^{2}$.

Based on the light band $C_{n}{ }^{2}$ model presented by ITU-R, microwave band $C_{n}{ }^{2}$ model may be given by the temperature of $T$, the humidity of $U$ as follows ${ }^{[1]}$ :

$$
\begin{gathered}
C_{n}^{2}=8.148 \times 10^{-56} v_{r m s} h^{10} \cdot e^{\frac{-h}{1000}}+2.7 \times 10^{-16} \cdot e^{\frac{-h}{1500}} \\
+C_{0} \cdot e^{\frac{-h}{100}}+6.4048 \times 10^{-12} \cdot h^{\frac{-11}{6}} N_{w}^{2} \\
N_{\text {wet }}=\frac{22814 U \exp (17.5 T / T+240.97)}{(T+273)^{2}}
\end{gathered}
$$

where, $C_{0}$ is $3.9 \times 10^{-12} \mathrm{~m}^{-2 / 3}, N_{\text {wet }}$ is the humidity in ITU R model. $T$ and $U$ vary with the elevation of $h$. According to the experimental results, we gain the expression of $T$ changed with height ${ }^{[1]}$ :

$$
T=t_{0}-0.006047 \times h(\cdot c)
$$

where $t_{0}$ is the ground temperature and its value is $20.85^{\text {c }}$. The expression of $U$ changed with height is ${ }^{[1]}$ :

$$
\mathrm{U}=\left\{\begin{array}{lc}
\mathrm{u}_{0}-0.0096 \mathrm{~h} & \mathrm{~h} \leq 5000 \\
28.8 & 5000<\mathrm{h} \leq 10000 \\
28.8-0.00556\left(\mathrm{~h}-10^{4}\right) & 10^{4}<\mathrm{h}<1.5 \times 10^{4}
\end{array}\right.
$$

where $u_{0}$ is the relative humidity of the ground and its value is $76.8 \%$.

\section{ATMOSPHERIC TURBULENCE GIVEN BY KOLMOGOROV MODEL}

Atmospheric refraction wave spectrum reflects the effect on signal caused by the atmospheric refractive. There are various forms of atmospheric refraction wave spectrum. In form of Kolmogorov model, it can be expressed as:

$$
\begin{aligned}
& \phi_{n}\left(k_{x}, k_{y}\right)=0.033 \times c_{n}^{2} \times e^{\left[-\frac{k_{x}^{2}+k_{y}^{2}}{k_{l}^{2}}\right]} \times\left[k_{x}^{2}+k_{y}^{2}+\frac{1}{L_{0}^{2}}\right]^{-\frac{11}{6}} \\
& \times\left[1+1.802 \sqrt{\frac{k_{x}^{2}+k_{y}^{2}}{k_{l}^{2}}}-0.254\left[\frac{k_{x}^{2}+k_{y}^{2}}{k_{l}^{2}}\right]^{\frac{7}{6}}\right]
\end{aligned}
$$

where $k_{X}{ }^{2}, k_{Y}{ }^{2}$ are frequency wave numbers on the $X$ direction and $Y$ direction respectively. $L_{0}{ }^{2}$ is the outer scale of turbulence, $l_{0}{ }^{2}$, the inner scale of turbulence, $k_{l}=3.3 / l_{0}$, $V_{r m s}$ is the RMS wind speed along the vertical path, its typical values is $21 \mathrm{~m} / \mathrm{s}$.

Phase spectrum can be represented by atmospheric refraction wave spectrum as follows ${ }^{[6][7]}$ :

$$
\phi\left(k_{x} k_{y}\right)=2 \pi\left(k_{x}^{2}+k_{y}^{2}\right) \cdot \Delta z \cdot \phi_{n}\left(k_{x}, k_{y}\right)
$$

where $\Delta z$ is the distance between adjacent phase screen on the wave propagation direction.

\section{THE INFLUENCE OF ATMOSPHERIC TURBULENCE ON THE VORTEX WAVE PHASE}

Radio waves propagating in the atmospheric space will be influenced by atmospheric turbulence ${ }^{[9]}$, then the signal phase will change. So we can't accurately discern the signal at the receiving end. This paper sets the outer-scale of atmospheric turbulence as $20 \mathrm{~m}$, the inner-scale as $0.005 \mathrm{~m}$. According to the $C_{n}{ }^{2}$ model of microwave band, we set $C_{n}{ }^{2}$ as $8 \times 10^{-15} \mathrm{~m}^{-2 / 3}$, the waist radius $W_{0}$ as $3 \mathrm{~mm}$, the electromagnetic wave frequency as $30 \mathrm{GHz}$, the number of grid points $N$ as 300 , the grid distance $\Delta x$ as $0.0005 \mathrm{~m}$. We study the vortex waves which OAM intrinsic value is 1 and set the transmission distance as $400 \mathrm{~m}$. The simulations results are as follows:

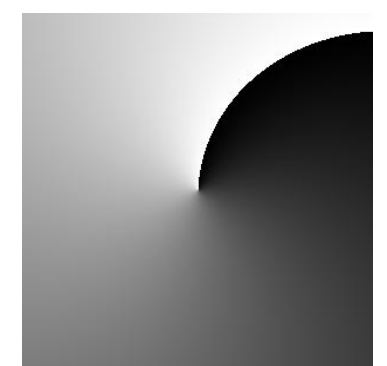

Figure 1(a)

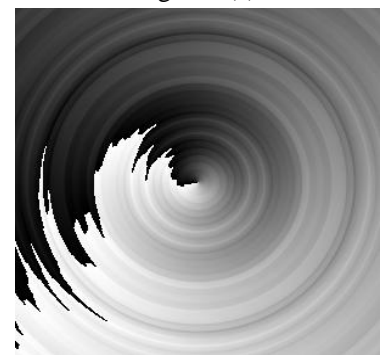

Figure 1(c)

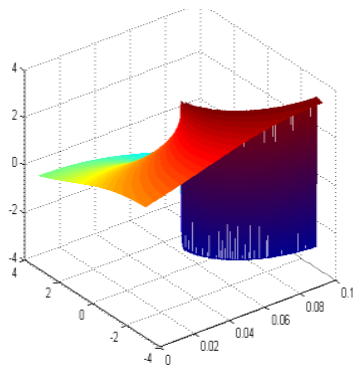

Figure 1(b)

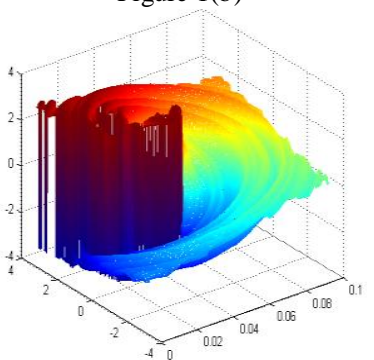

Figure 1(d)
Figure 1(a) is original signal phase; Figure1(b)is three dimensional graph of the original signal ; Figure1(c)is the variation of the signal phase which propagate through atmospheric turbulence; Figure1(d) is three dimensional graph of the signal which propagates through atmospheric turbulence

In the phase grayscale, the darker place represents the bigger phase value. By comparing Figure 1(a) and (c), we can clearly see the spiral phase changes obviously with a margin of about $37.5 \%$. In the three dimensional graph, height represents the value of signal phase, we can also see the change of signal phase. Then, we change the value of 
$C_{n}{ }^{2}$, when $C_{n}{ }^{2}$ is $8 \times 10^{-13} \mathrm{~m}^{-2 / 3}$, the simulation results are shown in Figure 2 (a) and 2(b).

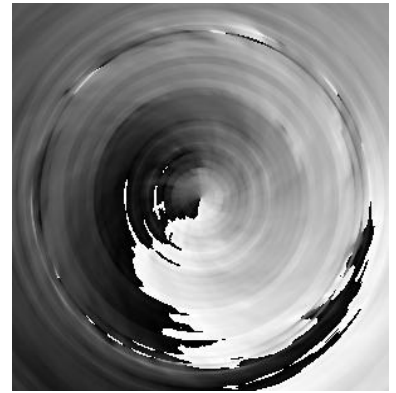

Figure 2(a)

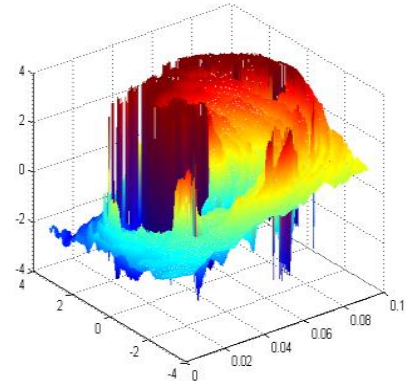

Figure 2(b)
Figure2(a) is the variation of the OAM phase when $\mathrm{C}_{n}{ }^{2}$ is $8 \times 10^{-13} \mathrm{~m}^{-2 / 3}$; Figure 2(b)is three-dimensional graph of the signal which propagates through turbulence with $\mathrm{C}_{\mathrm{n}}{ }^{2}$ of $8 \times 10^{-13} \mathrm{~m}^{-2 / 3}$

By comparing Figure 1 (c) with Figure 2 (a), it can be seen that the signal change is more intense. When $C_{n}{ }^{2}$ expands 100 times, phase fluctuation amplitude expand to $50 \%$. From the corresponding three-dimensional graph we can see that the surface is very uneven, so the spiral phase in different spatial orientation is affected differently.

On the basis of Figure 2, we change the transmission distance. We set the transmission distance as $1000 \mathrm{~m}$, the simulations results are as follows: Figure 3 (a) is the original signal phase grayscale,(b) is the corresponding three-dimensional graph, (c) is the grayscale of the signal which propagates through atmospheric turbulence,(d) is the corresponding three-dimensional graph.

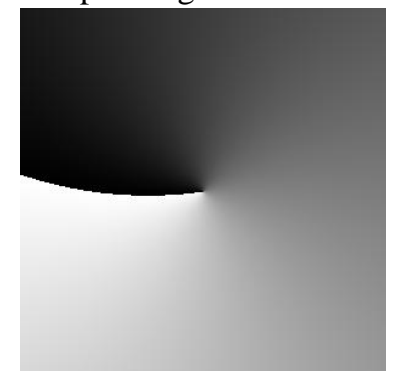

Figure3(a)

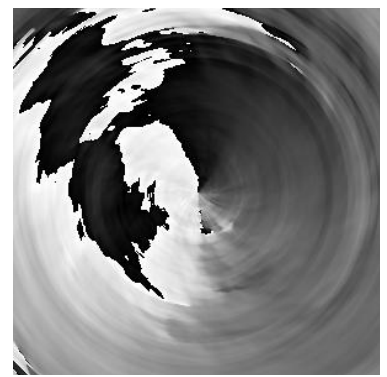

Figure3(c)

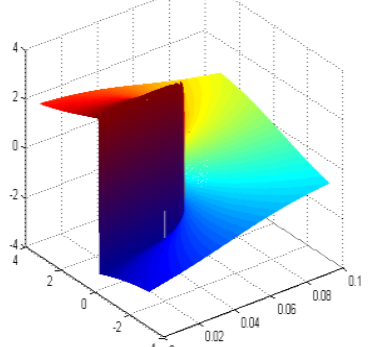

Figure3(b)

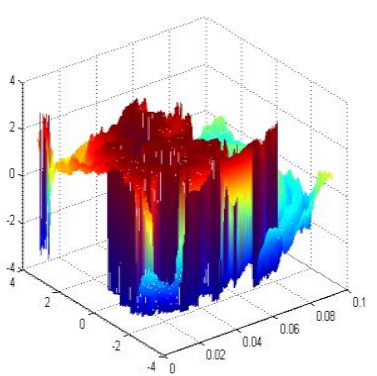

Figure3(d)
Figure3(a)is original signal phase grayscale; Figure3(b)is three dimensional graph of the original signal; Figure3(c)is the variation of the OAM phase when transmission distance is $1000 \mathrm{~m}$; Figure3(d)is three dimensional graph of the signal which propagates through a distance of $1000 \mathrm{~m}$
By comparing Figure 3(a) with 3(c), we can see the spiral phase change about $62.5 \%$. Compared with the situation that the transmission distance is $400 \mathrm{~m}$, the phase fluctuation is more serious. So the change of the transmission distance will cause the phase change.

Now, we alter $l$ values. When $l$ is $4, C_{n}^{2}$ is $8 \times 10^{-13} \mathrm{~m}^{-2 / 3}$ and transmission distance is $400 \mathrm{~m}$, the simulation results are as Figure 4(a) to (d).

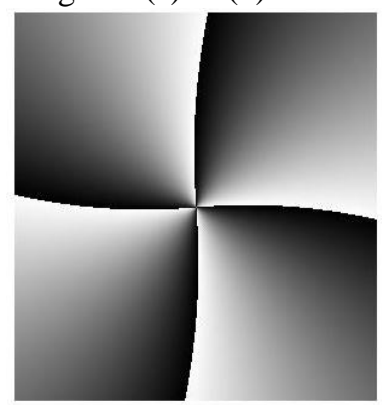

Figure 4(a)

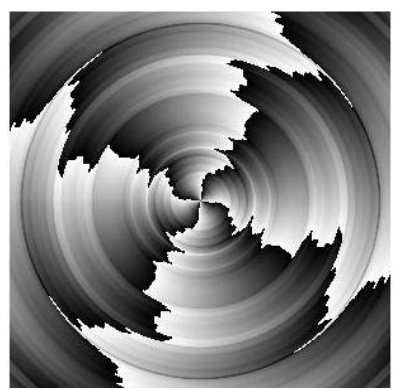

Figure 4(c)

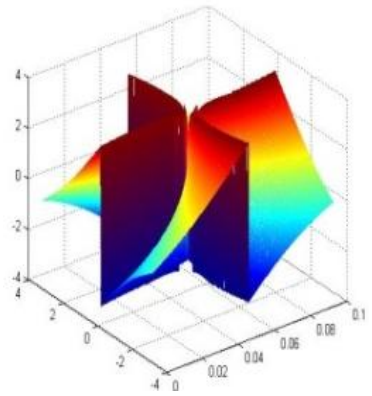

Figure 4(b)

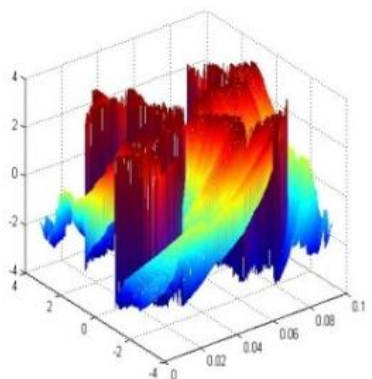

Figure 4(d)
Figure4(a)is original signal phase for $l=4$; Figure4(b)is three dimensional graph of the original signal for $l=4$; Figure $4(\mathrm{c})$ is the variation of the OAM phase when $\mathrm{C}_{\mathrm{n}}^{2}$ is $8 \times 10^{-13} \mathrm{~m}^{-2 / 3}$ for $l=4$; Figure $4(\mathrm{~d})$ is three dimensional graph of the signal which propagates through atmospheric turbulence for $l=4$.

With the same $l$ values and transmission distance, we change $C_{n}^{2}$, the simulation results are as Figure 5(a) and $5(\mathrm{~b})$.

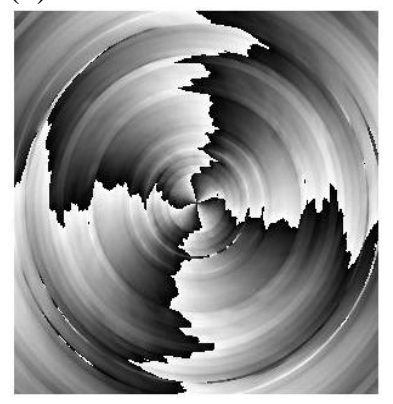

Figure 5(a)

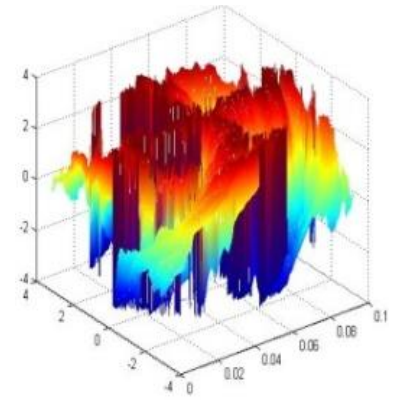

Figure 5(b)
Figure5(a) is the variation of the signal phase which propagate through atmospheric turbulence; Figure 5(b) is three dimensional graph of the signal which propagates through turbulence with $\mathrm{C}_{\mathrm{n}}{ }^{2}$ of $8 \times 10^{-13} \mathrm{~m}^{-2 / 3}$ for $l=4$

Then on the basis of Figure 5, we change transmission distance, the simulation results are as Figure 6. 


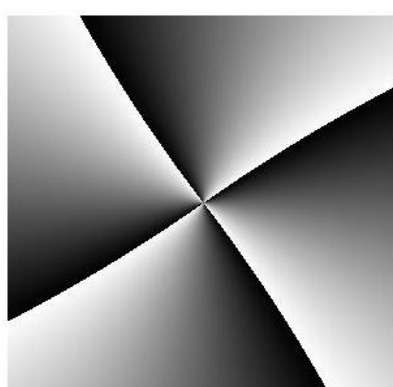

Figure 6(a)

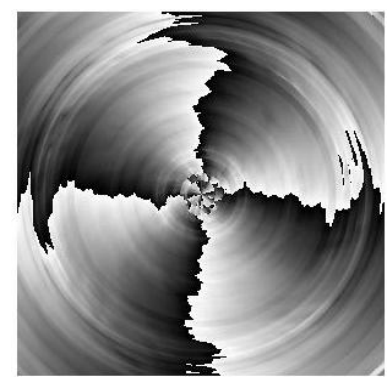

Figure 6(c)

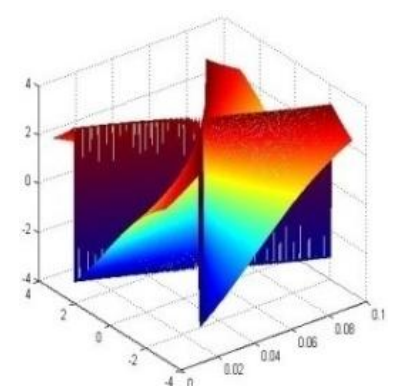

Figure 6(b)

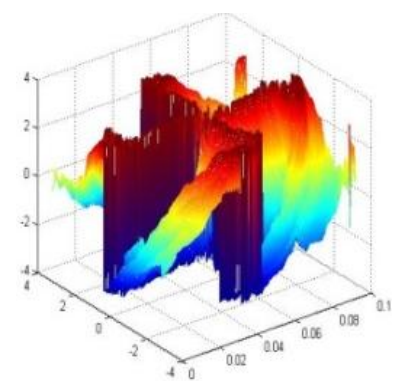

Figure 6(d)
Figure6(a) is original signal phase grayscale for $l=4$; Figure6(b) is three dimensional graph of the original signal for $1=4$; Figure $6(\mathrm{c})$ is the variation of the OAM phase when transmission distance is $1000 \mathrm{~m}$ for $l=4$; Figure6(d) is three dimensional graph of the signal which propagates through a distance of $1000 \mathrm{~m}$ for $l=4$;

From the above simulation results, we can draw a conclusion that $C_{n}{ }^{2}$ and transmission distance are factors which will cause the signal phase to change.

\section{CONCLUSION}

In this paper, we study the influence of atmospheric turbulence on the phase of vortex by simulation. We analyze the variations of vortex wave by changing the atmospheric refractive index structure constant and transmission distance. The results show that when atmosphere structure constant $C_{n}{ }^{2}$ increases, the signal change will increases. Similarly, when the transmission distance increases, the fluctuation of vortex phase will increases. It is important to know that the phase change of vortex wave passing through the atmospheric turbulence for discerning the correct signal at the receiving end. However, this paper focuses on studying the phase of the signal. Therefore, the future research will be comprehensive consideration of more parameters of the signal and how to reduce the influence of atmospheric turbulence on OAM communication system performance.

\section{ACKNOWLEDGEMENT}

This work is supported by National Natural Science Foundation of China (the Project 61271061).

\section{REFERENCES}

[1] YANG Ruike, WU Zhensen, ZHAO Zhenwei,. "Structure Constant Model of Troposphere Atmosphere Turbulence on Microwave Slant-Paths". Journal of Microwaves, vol. 12, pp. 64-68,June 2003.

[2] WEI Kejun, “Orbital angular momentum technology application in the wireless communication.Telecommunication" Network Technology, vol. 9, pp. 16-18,September 2013.

[3] ZOU Li, WANG Le ed. "Comenpensation of orbital-momentum multiplexed communication system with wavefront correction", Journal on Communication, vol. 10, pp. 76-84, October 2015.

[4] WANG Lijin, LI Qiang etc. "Numerical simulation and validation of phase screen distorted by atmospheric turbulence", Opto-Electronic Engineering, vol. 3, pp. 1-4, March 2007.

[5] ZHANG Huimin,LI Xinyang, "Numerical simulation of wavefront phase screen distorted by atmospheric turbulence". Opto-Electronic Engineering, vol. 33, pp.14-19, January 2006.

[6] ZOU Li, ZHAO Shengmei, WANG Le. "The Effects of Atmospheric Turbulence on the Orbital Angular Momentum-multiplexed System" . Acta Photonica Sinica, vol. 43, pp. 1-6, September 2014.

[7] ANDREWS L C, "Laser beam propagation through random media". Phillips R L.2rded.Bellingham,Washington:SPIE press, 2005, pp. 57-74.

[8] Sun Xue Hong,Li Qiang,Pang Dan Xu,Zeng Z hi Min. "New research progress of the orbital angular momentum technology in wireless communication: a survey ", Acta electronica Sinica, vol. 43, pp. 2306 - 2313,Nov 2015.

[9] Lin Le Ke,Zhang Xin ,Lu Chang Sheng,Li Hai Ying,Zhao Zhen Xiong. "Vortex communication and atmospheric turbulence",Chinese journal of radio science,vol.28 sup,pp.152-153,August 2013.

[10] Zhao Shengmei, Yang Hua ,Li Yong Qiang, Cao Fei,Sheng Yubo,Cheng Weiwen. "The influence of atmospheric on holographic ghost imaging using orbital angular momentum entanglement: Simulation and experimental studies", Optics Communications, vol. 294, pp.223-228,January 2013. 